\title{
Double differential neutron yields from thick targets used in space applications
}

\author{
Natalie A. McGirl ${ }^{1,}$, Luis A. Castellanos ${ }^{1}$, Ashwin P. Srikrishna ${ }^{1}$, Lawrence H. Heilbronn ${ }^{1}$, Chiara La Tessa $^{2}$, Adam \\ Rusek $^{2}$, Michael Sivertz ${ }^{2}$, Steve Blattnig ${ }^{3}$, Martha Clowdsley ${ }^{3}$, Tony Slaba ${ }^{3}$, and Cary Zeitlin ${ }^{4}$ \\ ${ }^{1}$ Department of Nuclear Engineering, University of Tennessee, 1004 Estabrook Rd, Knoxville, TN, USA, 37996 \\ ${ }^{2}$ NASA Space Radiation Laboratory, Brookhaven National Laboratory, Upton, NY, USA, 11973 \\ ${ }^{3}$ NASA, Langley Research Center, Hampton, VA, USA, 23681 \\ ${ }^{4}$ Lockheed Martin, Information Systems and Global Solutions, Houston, TX, USA, 77285
}

\begin{abstract}
In March 2016, secondary neutron production from thick-target shielding experiments were conducted at the National Aeronautics and Space Administration's (NASA) Space Radiation Laboratory at Brookhaven National Laboratory. Ion beams of proton, helium, and iron projectiles were aimed at aluminum targets with areal densities of 20,40 , and $60 \mathrm{~g} / \mathrm{cm}^{2}$. The ion beams were extracted at energies of 400 and $800 \mathrm{AMeV}$ and neutron yields were measured with liquid scintillators at $10^{\circ}$, $30^{\circ}, 45^{\circ}, 60^{\circ}, 80^{\circ}$, and $135^{\circ}$ off the beam axis. A second $60 \mathrm{~g} / \mathrm{cm}^{2}$ aluminum target was placed $3.5 \mathrm{~m}$ downstream from the middle of front target to study backscattered neutrons. Double differential thicktarget neutron yields for various combinations of projectile, projectile energy, target material, target thickness, and detector location were produced using the time-of-flight technique. These measurements will help NASA perform uncertainty analyses on their transport codes and contribute to shielding design studies for future space applications.
\end{abstract}

\section{Introduction}

The future of manned, deep-space missions includes the need to sufficiently protect astronauts from the ionizing radiation effects caused by Solar Energetic Particles (SEP) and Galactic Cosmic Rays (GCR) for an extended length of time. Habitats and other transit vehicles often contain areas of thick (30-40 g/ $\mathrm{cm}^{2}$ or greater) shielding materials such as aluminum or high density polyethylene (HDPE) [1]. While an enclosed, thickly-shielded environment may decrease an astronaut's exposure to the primary radiation field, the creation of a secondary radiation field, which includes neutrons and light charged ions, still poses a risk [2]. In particular, secondary neutrons are a concern due to their highly-penetrative nature and large dose equivalent conversion factors [3]. The purpose of this study is to determine the double differential thick-target yields for neutrons produced directly from GCR-like heavy ion interactions with aluminum or HDPE shielding. These results will be compared with transport model calculations and incorporated into the uncertainty analysis for transport codes developed by the National Aeronautics and Space Administration (NASA). This paper presents a selection of results from an experiment conducted in March 2016.

\section{Experiment Overview}

Secondary neutron production measurements for the 100hour March 2016 experiment occurred at Brookhaven
National Laboratory's (BNL) NASA Space Radiation Laboratory (NSRL). BNL's Booster accelerator was used to deliver the $400-$ and $800-\mathrm{MeV}$ protons, $400-\mathrm{AMeV}$ helium, and the 400 and $800-\mathrm{AMeV}$ iron beams to the 20 , 40 , and $60 \mathrm{~g} / \mathrm{cm}^{2}$ thick aluminum targets $\left(100 \times 30 \mathrm{~cm}^{2}\right)$. A back aluminum target with an areal density of $60 \mathrm{~g} / \mathrm{cm}^{2}$ $\left(100 \times 100 \times 22.2 \mathrm{~cm}^{3}\right)$ was placed $3.5 \mathrm{~m}$ downstream from the middle of the front target. The addition of this target allowed for the study of neutrons scattered from or produced in the back target, which will occur at a later date. Additionally, two beam-defining EJ-228 plastic scintillator detectors were placed directly in front of the forward target and identified valid beam particles from coincidence events.

Neutrons were detected with three EJ-301 liquid scintillators placed at $10^{\circ}, 30^{\circ}, 45^{\circ}$, and three EJ-309 liquid scintillators placed at $60^{\circ}, 80^{\circ}$, and $135^{\circ}$ off the beam axis, with flight paths ranging from 2 to $3 \mathrm{~m}$ from the center of the front target to the front face of the liquid scintillator. Each liquid scintillator was $12.7 \mathrm{~cm}$ in diameter and $12.7 \mathrm{~cm}$ tall. Two thin EJ-304 solid plastic scintillators $\left(12.7 \times 12.7 \times 0.635 \mathrm{~cm}^{3}\right)$ covered the front face of each liquid scintillator to distinguish between incident neutral and charged particle events. Finally, a shadow bar system was used to allow for full background characterization. This system consisted of two iron bars with lengths of 1 and $2 \mathrm{~m}$. These shadow bars fully blocked the front face of a liquid scintillator and prevented neutrons originating directly from the front target from entering the scintillator. Liquid scintillator

\footnotetext{
* Corresponding author: nmcgirl@vols.utk.edu
} 
locations, target locations, and flight path values are shown in Figure 1.

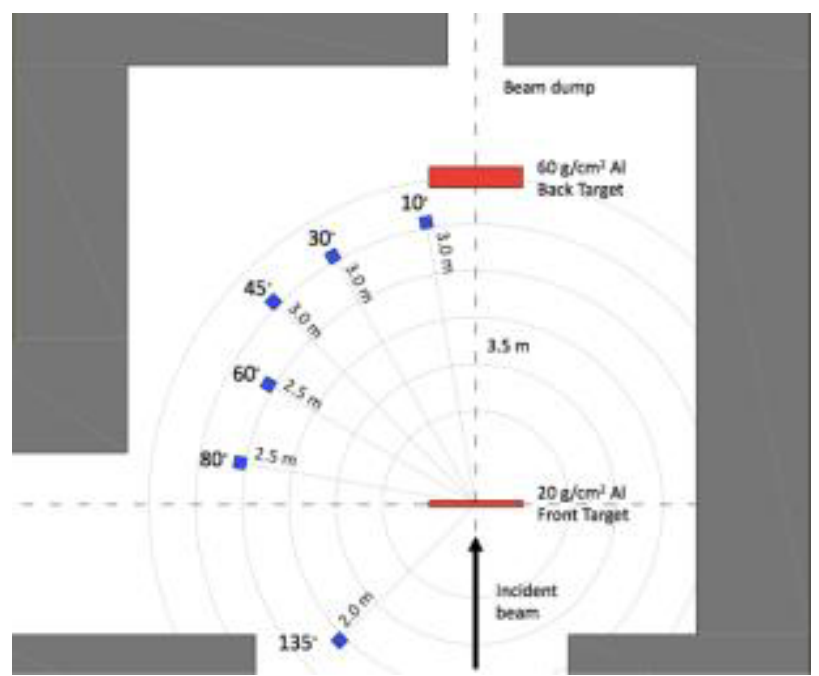

Fig. 1. March 2016 NSRL target room experimental setup for $20 \mathrm{~g} / \mathrm{cm}^{2}$ front and $60 \mathrm{~g} / \mathrm{cm}^{2}$ back aluminum targets. Grey blocks represent concrete walls.

\section{Neutron Time of Flight Analysis}

Several steps were taken to determine the double differential thick-target neutron yields for the March 2016 100-hour experiment. First, the charge deposited in the beam-defining "start" scintillators were plotted against each other. A noticeable beam spot was identified and a graphical ROOT cut was taken around it [4]. This ensured that only beam particles that did not deviate too far from the center of the beam were taken as source particles incident upon the front target. On average, approximately $30 \%$ of the beam particles were eliminated from the analysis with the good beam cut.

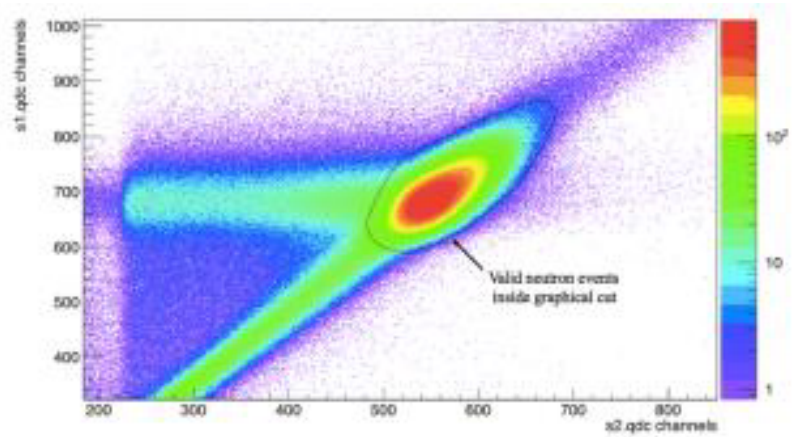

Fig. 2. Charge deposited in start scintillators for a 400-AMeV iron beam with good beam cut boundaries displayed.

Next, the neutral and charged particle events that set the liquid scintillator trigger were gated on in ROOT by selecting the self-time peak in the time-to-digital conversion (TDC) spectrum. The contributions to the liquid scintillator signals were then separated using the thin EJ-304 "veto" detectors located in front of the scintillators. Events that registered in both the veto and liquid scintillator detectors were taken to be the result of a charged particle interaction, due to the low probability of a neutron or gamma interaction in the veto detector [5].
By examining the veto detector's charge-to-digital converter (QDC) output, the neutral and charged particle information was separated and the neutral particle data were refined further to determine the neutron and gamma signal contributions.

Pulse shape discrimination (PSD) was used to separate neutrons and gammas based on the differences between the charge contained in the first 20 to $35 \mathrm{~ns}$ of a signal (h_qde) and in the total signal (t_qde) [6]. An example of a typical PSD plot is shown in Figure 3. The lowest energy of neutron-gamma separation was examined by taking one-channel-wide slices of the PSD plots and projecting them onto the $\mathrm{Y}$-axis. The resulting neutron and gamma peaks were considered well-separated if their $2 \sigma$ values did not overlap. The channel number at the separation threshold was converted to incident neutron energy $(\mathrm{MeV})$ using the experimentally determined calibration curves of the liquid scintillators and a light output calibration [7]. Because a neutron may deposit all of its energy in a single interaction, the energy at the separation threshold represented the lowest energy neutron detectable by the scintillator. A $4.0 \mathrm{MeV}$ neutron was detected at the separation threshold for the scintillators at $10^{\circ}, 30^{\circ}$, and $45^{\circ}$, while a $2.0 \mathrm{MeV}$ neutron was detected at the separation threshold for the scintillators at $60^{\circ}, 80^{\circ}$, and $135^{\circ}$. Afterwards, neutron and gamma event data were extracted from the PSD plots using ROOT graphical cuts.

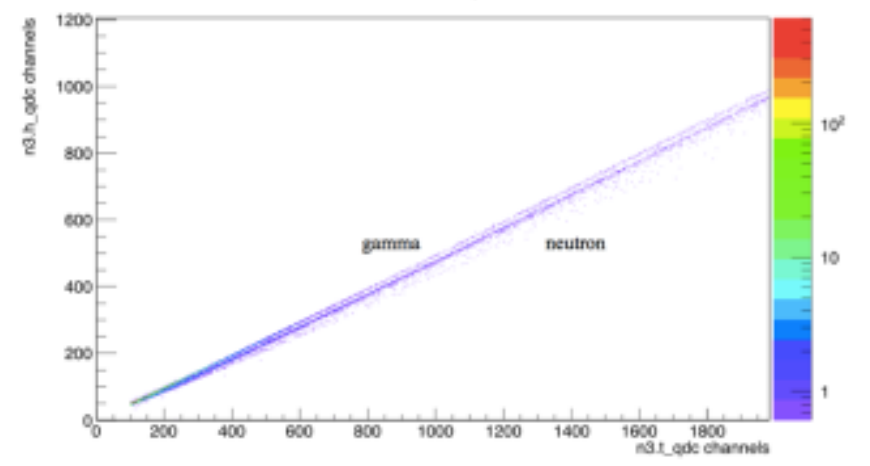

Fig. 3. Neutron and gamma PSD at $45^{\circ}$ for $400-\mathrm{MeV}$ protons on 20,40 , and $60 \mathrm{~g} / \mathrm{cm}^{2}$ aluminum front targets; $60 \mathrm{~g} / \mathrm{cm}^{2}$ back aluminum target.

To convert the start scintillator's time-to-digital converter (TDC) outputs to neutron energy spectra, the time-of-flight (TOF) method was utilized. TDC spectra collection started with a coincidence between the beamdefining scintillators and a neutron detector, and stopped once a delayed signal from the beam-defining scintillators was registered. To determine the neutron times of flight, a reference channel number, which corresponded to the gammas that were created in the projectile-target interactions and subsequently detected in the liquid scintillators, was identified on the TDC spectra. This channel number was found by plotting the TDC spectra gated on the gammas identified in PSD. The centroid of the resulting prompt gamma peak was used as the reference channel number, which was different for each beam-target combination. 
Before the neutron TOF could be calculated, the background neutron contributions were taken into consideration. During the experiment, multiple runs were taken for each beam-target configuration with and without a shadow bar covering the front face of the liquid scintillators. Because runs varied in duration, system live time, and source particle exposure, counts $\left(N_{\text {measured }}\right)$ in each covered run were combined and normalized to the recorded number of source particles $\left(s_{1}: s_{2}\right)$, good beam fraction $(G B)$, and live time $(L T)$ per equation 1 . This process was then repeated for the uncovered runs. After normalization, the sum of the covered runs for a given beam-target combination were subtracted from the sum of the uncovered runs. This meant the final spectra only contained information for neutrons coming directly from the front target, and not background neutrons that entered from the sides of the liquid scintillator or background neutrons that bypassed the shadow bars by skipping off the floor and entered the front of the scintillator.

$$
\text { Yield }=\frac{\sum_{i=1}^{n} \frac{N_{i(\text { measured })}}{L T_{i}}}{\sum_{i=1}^{n} G B_{i} \times\left(s_{1} \cdot s_{2}\right)_{i}}
$$

Neutron times of flight $\left(t_{n}\right)$, velocities $\left(\beta_{n}=v_{n} / c\right)$, and kinetic energies $\left(T_{n}\right)$ were calculated using equations 2 through 5 , respectively. The TDC calibration $(k)$ varied from $0.228 \mathrm{~ns} /$ channel to $0.238 \mathrm{~ns} /$ channel, depending upon the liquid scintillator. Neutron flight paths $(d)$ also varied depending upon the liquid scintillator. The prompt gamma peak channel number $\left(t d c_{g}\right)$ was dependent upon the beam-target combination, while gamma velocity $(c)$ and the neutron rest mass $\left(m_{n}\right)$ were constant values. Finally, the TDC channel number of neutron events $\left(t d c_{n}\right)$ were taken from the TDC output spectra.

$$
\begin{gathered}
t_{n}=(d / c)+k\left(t d c_{g}-t d c_{n}\right) \\
v_{n}=d / t_{n} \\
T_{n}=(\gamma-1) m_{n} \\
\gamma=\left(1-\beta_{\mathrm{n}}^{2}\right)^{-1}
\end{gathered}
$$

After converting channel number to neutron energy, an energy-dependent neutron detection efficiency correction factor was applied to each bin. Efficiencies were calculated for a $12.7 \mathrm{~cm}$ in length, $12.7 \mathrm{~cm}$ in diameter cylindrical liquid scintillator placed 2.0, 2.5, and $3.0 \mathrm{~m}$ away from a neutron source using the SCINFULQMD Monte Carlo code [8]. Double differential thicktarget neutron yields were then calculated by dividing the efficiency-adjusted neutron yields by the solid angle of the liquid scintillator and the energy bin width for units of neutrons per source particle per steradian per energy (neutrons / S.P. / $\Omega$ / MeV).

Finally, the uncertainties in the counts were taken into consideration. Statistical uncertainties were calculated in ROOT and varied for each bin. Systematic uncertainties included uncertainties in solid angle, neutron detection efficiency, and graphical cuts. Solid angle uncertainties varied from $6.5 \%$ to $13.8 \%$ depending upon the flight path of the liquid scintillator and the front target thickness. The uncertainty in neutron detection efficiency was estimated at $15 \%$ using published experimental and SCINFULQMD calculated results [8]. Finally, the uncertainty in the ROOT graphical cuts was estimated at 5\%. The summary of these systematic uncertainties organized by front target thickness is included in Table 1.

Table 1. Fractional systematic uncertainties for front aluminum target thicknesses of 20,40 , and $60 \mathrm{~g} / \mathrm{cm}^{2}$.

\begin{tabular}{|c|c|c|c|}
\hline \multirow{2}{*}{ Type } & \multicolumn{3}{|c|}{$\begin{array}{c}\text { Fractional } \\
\text { at } \mathbf{1 0}^{\circ}, \mathbf{1 3 5}^{\circ}\end{array}$} \\
\cline { 2 - 4 } & $\mathbf{2 0} \mathbf{g} / \mathbf{c m}^{\mathbf{2}}$ & $\mathbf{4 0} \mathbf{g} / \mathbf{c m}^{\mathbf{2}}$ & $\mathbf{6 0} \mathbf{g} / \mathbf{c m}^{\mathbf{2}}$ \\
\hline Solid Angle & $6.5,8.7 \%$ & $8.9,11.3 \%$ & $11.4,13.8 \%$ \\
\hline $\begin{array}{c}\text { Detection } \\
\text { Efficiency }\end{array}$ & \multicolumn{3}{|c|}{$15 \%$} \\
\hline $\begin{array}{c}\text { ROOT } \\
\text { Graphical Cuts }\end{array}$ & \multicolumn{3}{|c|}{$5 \%$} \\
\hline
\end{tabular}

\section{Selection of Experimental Results}

A selection of background-subtracted results from the March 2016 experiment are provided below in Figures 4 through 8. Each figure contains double differential thicktarget neutron yields at $10^{\circ}$ and $135^{\circ}$ for a single beam species on the 20,40 , and $60 \mathrm{~g} / \mathrm{cm}^{2}$ front aluminum targets. The $10^{\circ}$ yields were multiplied by a factor of 100 to prevent the overlap of the $10^{\circ}$ and $135^{\circ}$ low-energy yields. Only statistical uncertainties were shown and they varied widely depending upon the ion beam. In general, statistical uncertainties from this experiment decreased with an increase in front target thickness. As the front target thickness increased, the number of nuclear interactions between the incident beam and target material also increased, resulting in a larger production of secondary neutrons. Additionally, an increase in beam energy typically led to lower statistical uncertainties because of the increased number of beam-target interactions. However, this was not seen with the 800$\mathrm{AMeV}$ iron beam, as discussed later. Statistical uncertainties also improved as the neutron energy increased for the $10^{\circ}$ liquid scintillator, but the uncertainties worsened at the highest neutron energies (about 60 to $90 \mathrm{MeV}$ ) for the $135^{\circ}$ liquid scintillator.

Double differential thick-target neutron yields at $10^{\circ}$ mostly consisted of neutrons produced during projectile breakup. The characteristic forward angle peak was located at approximately $70 \%$ of the incident beam energy and the yields rapidly decreased after this point, as expected. The thick-target neutron measurements and uncertainties at $10^{\circ}$ suffered from background subtraction at low energies due to the liquid scintillator's proximity to the back target. Neutrons produced by other secondary ions or reflected off the back target typically had energies less than $100 \mathrm{MeV}$. These neutrons entered through the sides of the $10^{\circ}$ liquid scintillator instead of the front face, which meant they were tagged as background events during the shadowed runs. Additionally, many of the 
beams were high enough in energy to punch through the front target, which resulted in secondary neutron production by primary beam interactions in the back target. The higher than expected background yields below $100 \mathrm{MeV}$ resulted in lower-than-expected backgroundsubtracted yields as well as poor statistics from error propagation. While the average fractional uncertainties below $100 \mathrm{MeV}$ varied widely depending upon the ion beam, all average uncertainties above $100 \mathrm{MeV}$ were less than $20 \%$.

Yields at $135^{\circ}$ were dominated by low-energy, isotropically emitted neutrons from target evaporation. Detected neutrons at this angle typically had an energy less than $100 \mathrm{MeV}$ and yields exponentially decreased with energy. Statistical uncertainties were best for neutron measurements at $135^{\circ}$ when compared to the other liquid scintillator locations due to the low neutron background at back angles. The average fractional uncertainties for all beams at all neutron energies ranged from $3.5 \%$ to $10 \%$.

Double differential thick-target neutron yields for the $400-\mathrm{AMeV}$ iron beam are displayed in Figure 4.
This beam was stopped in all aluminium target thicknesses, indicating that only secondary particles were emitted from the front target. At $10^{\circ}$, the average fractional statistical uncertainties below $100 \mathrm{MeV}$ for the 20,40 , and $60 \mathrm{~g} / \mathrm{cm}^{2}$ thick beam-target configurations were $12 \%, 9.5 \%$, and $9.0 \%$, respectively. Above 100 $\mathrm{MeV}$, fractional uncertainties were less than 3.5\%.

Figure 5 contains double differential yields for 800 $\mathrm{AMeV}$ iron on 20,40 , and $60 \mathrm{~g} / \mathrm{cm}^{2}$ aluminum front targets. Yields generated with the $800-\mathrm{AMeV}$ iron beam were generally higher than with the $400-\mathrm{AMeV}$ iron beam, except with the $20 \mathrm{~g} / \mathrm{cm}^{2}$ beam-target configuration. The $800-\mathrm{AMeV}$ iron beam stopped in the 40 and $60 \mathrm{~g} / \mathrm{cm}^{2}$ aluminum targets, but punched through the $20 \mathrm{~g} / \mathrm{cm}^{2}$ target. This meant that the incident ion beam also interacted in the back $60 \mathrm{~g} / \mathrm{cm}^{2}$ aluminum target, increasing the number of neutrons that entered through the sides of the liquid scintillator at $10^{\circ}$. Double differential yields at $20 \mathrm{~g} / \mathrm{cm}^{2}$ were lower than expected for all secondary neutron energies because of this increase in the "neutron background" close to the back target.
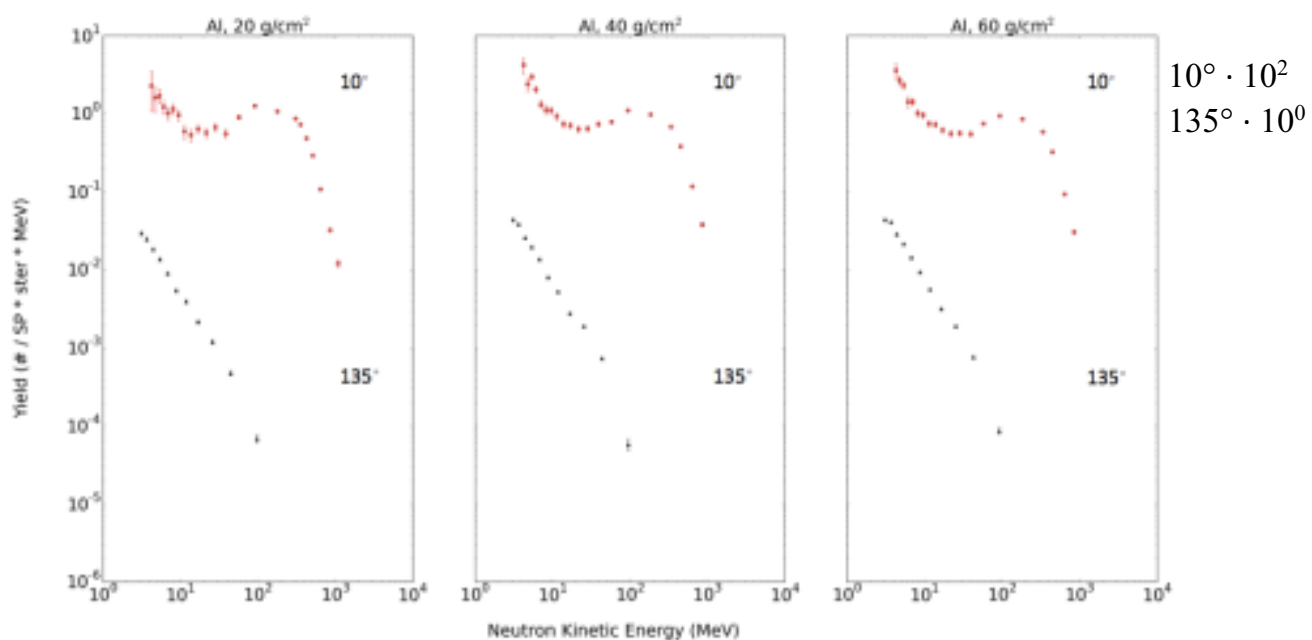

Fig. 4. Background subtracted double differential thick-target neutron yields at $10^{\circ}$ and $135^{\circ}$ for $400-\mathrm{AMeV}$ iron projectiles on 20 , 40 , and $60 \mathrm{~g} / \mathrm{cm}^{2}$ front and $60 \mathrm{~g} / \mathrm{cm}^{2}$ back aluminum targets. The $10^{\circ}$ yields were multiplied by a factor of 100 to prevent overlap.
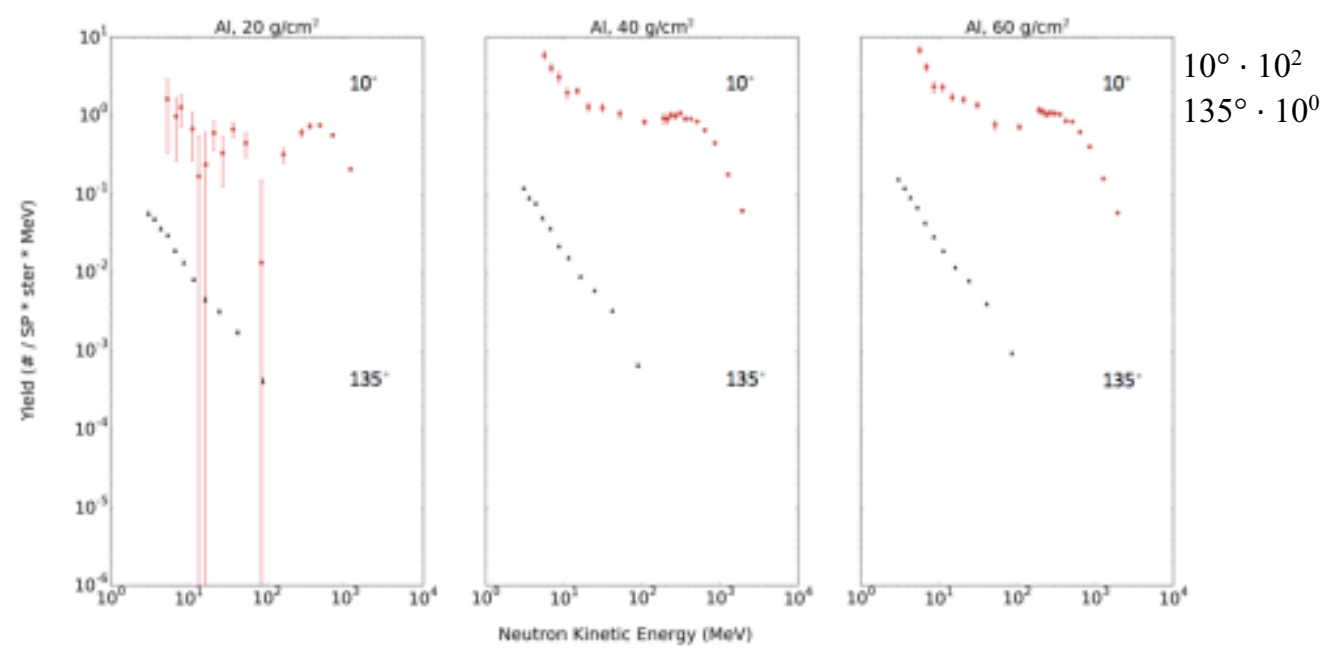

Fig. 5. Background subtracted double differential thick-target neutron yields at $10^{\circ}$ and $135^{\circ}$ for $800-\mathrm{AMeV}$ iron projectiles on 20 , 40 , and $60 \mathrm{~g} / \mathrm{cm}^{2}$ front and $60 \mathrm{~g} / \mathrm{cm}^{2}$ back aluminum targets. The $10^{\circ}$ yields were multiplied by a factor of 100 to prevent overlap. 
Adding the shadowed and unshadowed uncertainties in quadrature resulted in average uncertainties over $100 \%$ below $100 \mathrm{MeV}$. However, the average uncertainty was less than $8.5 \%$ above neutron energies of $100 \mathrm{MeV}$. For the 40 and $60 \mathrm{~g} / \mathrm{cm}^{2}$ target, the average uncertainties below $100 \mathrm{MeV}$ were less than $13 \%$, and less than 5\% at higher energies.

Double differential neutron yields for the 400- and $800-\mathrm{MeV}$ proton beams are displayed in Figures 6 and 7. Both beams punched through all front target thicknesses and interacted in the back target, resulting in poor statistics below $100 \mathrm{MeV}$, similar to what was seen with the $800-\mathrm{AMeV}$ iron beam on $20 \mathrm{~g} / \mathrm{cm}^{2}$ aluminum. Due to the increase in beam-target interactions and subsequent increase in secondary neutrons with a higher beam energy, average uncertainties were lower for the 800-
$\mathrm{MeV}$ proton system at all target thicknesses when compared with the $400-\mathrm{MeV}$ proton system. Depending upon the target thickness, average uncertainties below 100 $\mathrm{MeV}$ for the $400-$ and $800-\mathrm{MeV}$ proton beams ranged from $32 \%$ to $54 \%$ and $15 \%$ to $31 \%$, respectively. Above $100 \mathrm{MeV}$, average uncertainties were less than $22 \%$ (400$\mathrm{MeV}$ protons) and less than $10 \%(800-\mathrm{MeV}$ protons $)$.

Finally, the 400-AMeV helium beam-target double differential yields are displayed in Figure 8. While the helium beam also punched through all target thicknesses, the uncertainty issues below $100 \mathrm{MeV}$ were less pronounced than what was seen with the proton beams and the $800-\mathrm{AMeV}$ iron beam on $20 \mathrm{~g} / \mathrm{cm}^{2}$ aluminum. Average uncertainties below $100 \mathrm{MeV}$ ranged from $12 \%$ to $27 \%$, depending upon the target thickness, while uncertainties above $100 \mathrm{MeV}$ were less than $5 \%$.
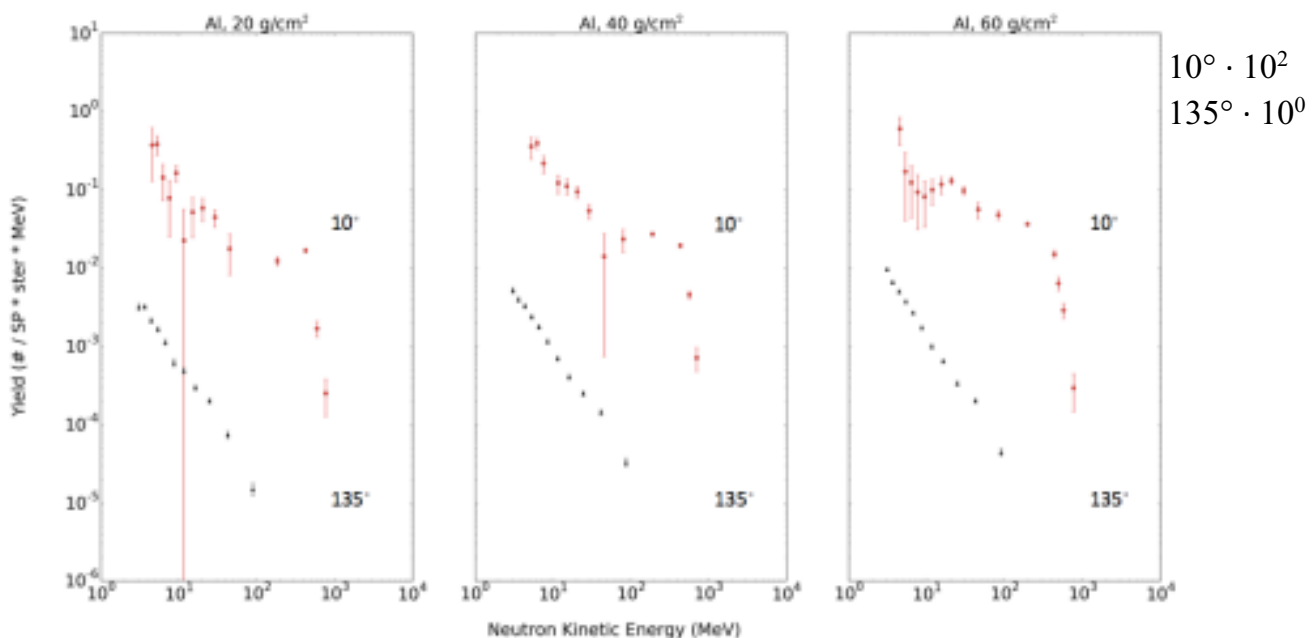

Fig. 6. Background subtracted double differential thick-target neutron yields at $10^{\circ}$ and $135^{\circ}$ for $400-\mathrm{MeV}$ proton projectiles on 20 , 40 , and $60 \mathrm{~g} / \mathrm{cm}^{2}$ front and $60 \mathrm{~g} / \mathrm{cm}^{2}$ back aluminum targets. The $10^{\circ}$ yields were multiplied by a factor of 100 to prevent overlap.
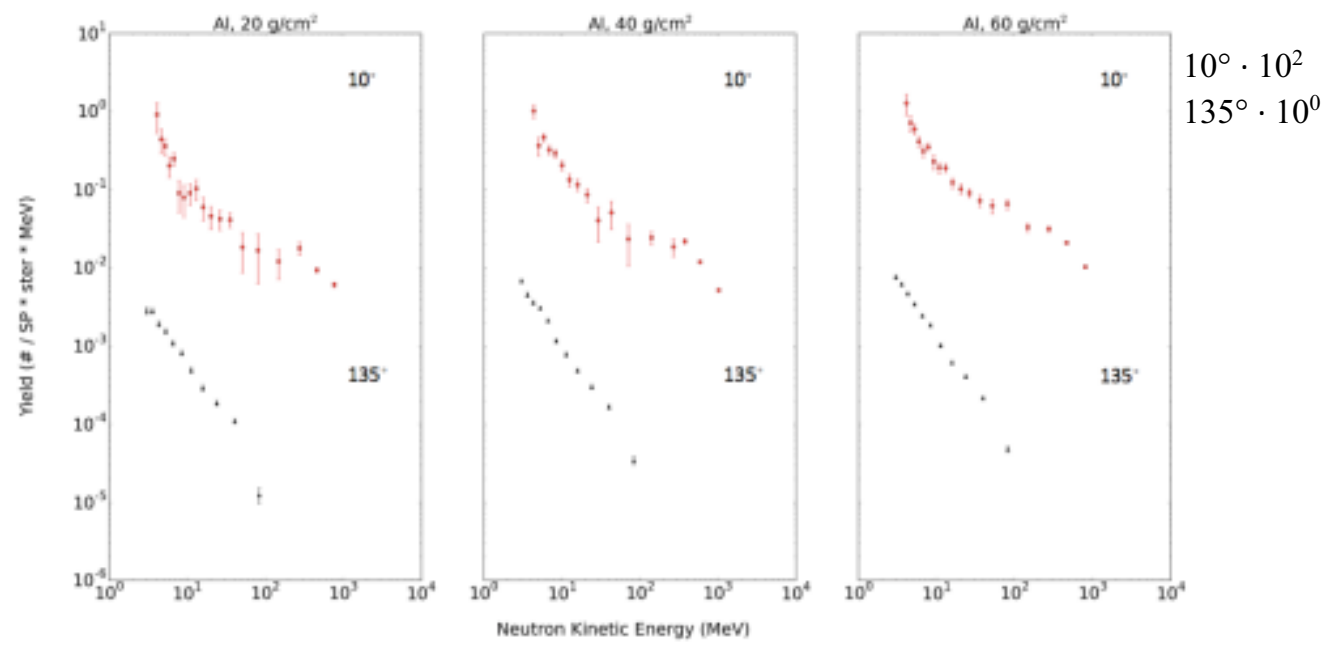

Fig. 7. Background subtracted double differential thick-target neutron yields at $10^{\circ}$ and $135^{\circ}$ for $800-\mathrm{MeV}$ proton projectiles on 20 , 40 , and $60 \mathrm{~g} / \mathrm{cm}^{2}$ front and $60 \mathrm{~g} / \mathrm{cm}^{2}$ back aluminum targets. The $10^{\circ}$ yields were multiplied by a factor of 100 to prevent overlap. 

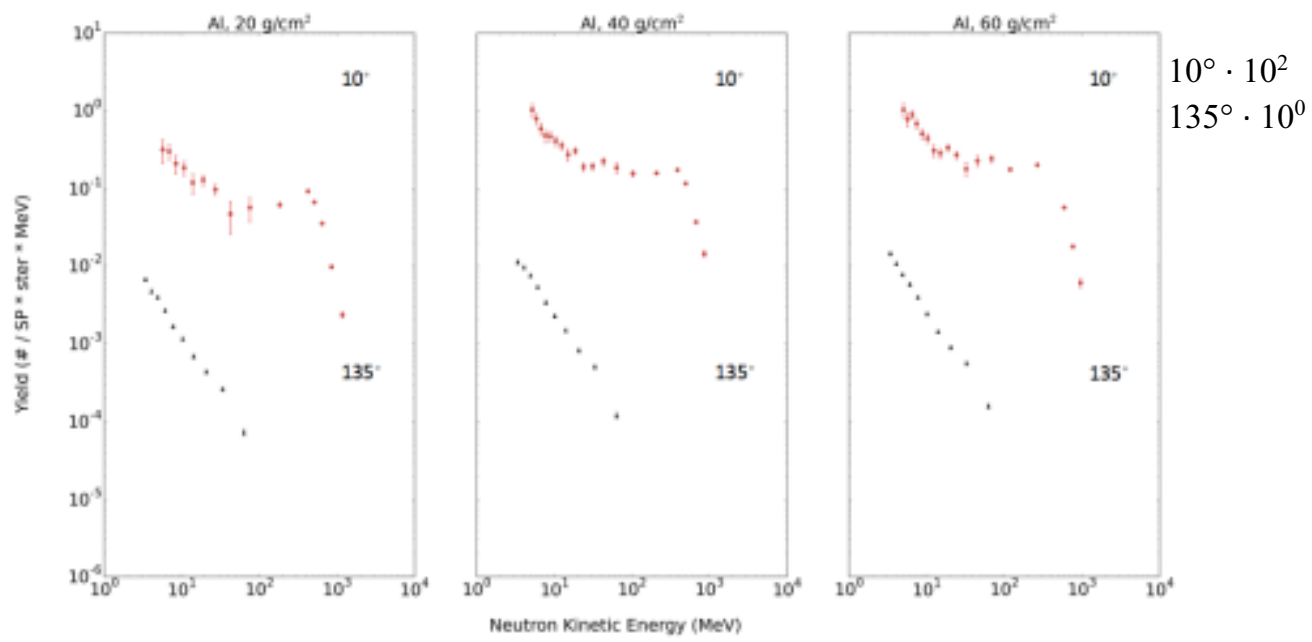

Fig. 8. Background subtracted double differential thick-target neutron yields at $10^{\circ}$ and $135^{\circ}$ for $400-\mathrm{AMeV}$ helium projectiles on 20 , 40 , and $60 \mathrm{~g} / \mathrm{cm}^{2}$ front and $60 \mathrm{~g} / \mathrm{cm}^{2}$ back aluminum targets. The $10^{\circ}$ yields were multiplied by a factor of 100 to prevent overlap.

\section{Future Work}

Further analysis will include quantifying the energy resolution of each neutron event using the method described in [9]. ROOT graphical cut uncertainties will be improved by reanalyzing the data with new graphical cuts. A fit to the background data at the $10^{\circ}$ liquid scintillator will be considered to improve statistical uncertainties below $100 \mathrm{MeV}$. Additionally, these experimental results will be compared to MCNP-, PHITS-, and GEANTsimulated double differential thick target yields and their differences will be explored. In addition to this experimental dataset, two 200-hour experiments are scheduled for late 2016 and 2017 at BNL's NSRL. These experiments will follow the same setup and execution of the March 2016 experiment, but will test different beamtarget configurations. Targets considered will include 20, 40 , and $60 \mathrm{~g} / \mathrm{cm}^{2}$ aluminum and HDPE targets, and ion beams will include proton, helium, carbon, silicon, and iron projectiles at $0.4,0.8,1.0$, and $2.5 \mathrm{AGeV}$. The analysis procedure laid out in this paper will be repeated for the new datasets.

\section{Summary}

100 hours of thick-target experiments were conducted in March 2016 at BNL's NSRL facility. Five ion beam species (400- and $800-\mathrm{AMeV}$ iron, 400 and $800-\mathrm{MeV}$ protons, and $400-\mathrm{AMeV}$ helium) were bombarded on three aluminum upstream targets of varying areal thicknesses $\left(20,40\right.$, or $\left.60 \mathrm{~g} / \mathrm{cm}^{2}\right)$. A second $60 \mathrm{~g} / \mathrm{cm}^{2}$ aluminum target was placed $3.5 \mathrm{~m}$ downstream from the front target. Neutrons and light charged ions were measured at six angular locations off beam axis using EJ301 and EJ-309 liquid scintillators. Neutron events was separated from the gamma and light charged ion events using a series of cuts in ROOT and then normalized to the number of source particles. Data was then background subtracted for each beam-target configuration, and the TDC channels were converted to energy using the time- of-flight method. Finally, neutron yields were corrected for neutron detection efficiency and then normalized to solid angle and energy bin width to produce double differential thick-target yields in units of neutrons / source particle / steradian / MeV. Neutron yields at $10^{\circ}$ suffered from a high neutron background due to the liquid scintillator's proximity to the back target, while yields at $135^{\circ}$ had the best uncertainties of all the angles. Additional analysis is needed to explore other methods of quantifying background neutrons at $10^{\circ}$, as well as to compare the experimental results presented here to Monte Carlo simulations. Ultimately, the experimental double differential thick target yields will be incorporated into NASA's uncertainty analysis for their radiation transport codes.

This work was supported by Grants NNX12AL51A and NNX15AD89A from the United States National Aeronautics and Space Administration. Special thanks to Matthew Beach, Ph.D. for his assistance with ROOT. Additional thanks to the team at Brookhaven National Laboratory for their extensive assistance with the setup and execution of the experiment.

\section{References}

1. P.-E. Tsai, et. al., 2015 IEEE Aerospace Conference, 1-7 (2015).

2. L. Heilbronn, et. al., Nucl. Sci. Eng., 132, 1-15 (1999).

3. P.-E. Tsai, "Study of secondary particles produced from heavy-ion interactions," Ph.D. dissertation, Dept. of Nucl. Eng., Univ. of TN, Knoxville, TN (2015).

4. R. Brun, F. Rademakers, Nucl. Instr. Meth. Phys. Res. A, 389, 81-86 (1997).

5. T. Nakamura, L. Heilbronn, Handbook on Secondary Particle Production and Transport by High-Energy Heavy Ions (Singapore: World Scientific Publishing Co. Pte. Ltd., 2006). 
6. J. H. Heltsley, et. al., Nucl. Instr. Meth. Phys. Res. A, 263, 441-445 (1988).

7. V. V. Verbinski, et. al., Nucl. Inst. Meth., 65, 8-25 (1968).

8. D. Satoh, et. al., J. Nucl. Sci. Tech., 39, 657-660 (2002).

9. Y. Iwata, et. al., Phys. Rev. C, 64 (2001). 